Onkologie 1996;19:110-112

\title{
Contents, Vol. 19, No. 2, 1996
}

\section{Inhalt}

Review Articles

114 Tumor Immunotherapy with Bispecific Antibodies

Hartmann, E; Renner, C; Pfreundschuh. M. (Homburg/Saar)

119 Neuroendocrine Tumors of the Gastroenteropancreatic System: I. Diagnostic Advances

Scherübl, H.; Faiss, S.; Zimmer, T.; Riecken, E.-O.; Wiedenmann, B. (Berlin)

126 Biochemical Markers of Bone Remodelling

Engler, H.; Thürliraann, B.; Riesen, W.F. (St. Gallen)

132 How Does Diet Contribute to Colon Cancer Development? Interaction between Genetic

Alterations and Nutrients in the Deregulation of Growth

Marian, B. (Wien)

Original Articles

140 Levamisole and 5-Fluorouracil as an Adjuvant Therapy for Patients after Curative Resection of Colon Carcinoma Dukes' Stage C (TNM HI): More Disadvantages than Advantages

Wassner, A.; Heidemann, E. (Stuttgart)

147 Combined Locoregional and Systemic Adjuvant

Chemotherapy of Stage II and III Rectal Carcinoma

Kornek, G.V. (Wien); Depisch, D. (Wr. Neustadt); Salem, G. (St. Pollen); Karall, M. (Wr.

Neustadt); Rohrbacher, M. (St. Pölten): Scheithauer, W. (Wien)

153 A Phase I Dose Escalation Trial of Intravenous Treosulfan in Refractory Cancer

Harstrick, A.; Wilke, H.; Eberhardt, W.; Klaassen, U.; Strumberg, D.;

Korn, M,; Scheulen, M.E. (Essen); Baumgart, J. (Hamburg); Seeber, S. (Essen)

159 Treatment of Adult Metastatic Soft-Tissue Sarcoma with Doxorubicin/Ifosfamide: Better

Hematoiogic Tolerance by G-CSF?

Weh, H.J.; de Wit, M.; Zornig, C; Hossfeld, D.K. (Hamburg)

164 Placebo-Controlled Trial of Medroxyprogesterone

Acetate in Gastrointestinal Malignancies and Cachexia

Kornek, G.V.; Schenk, T.; Ludwig, H.; Hejna, M; Scheithauer, W. (Wien)

170 Comparison of the Clinical Relevance of CE A, CA 15-3 and MCA in Breast Cancer

Bremer, K.; Micus, S.; Bremer, G (Bochum); Eberhard, A. (Dortmund)

176 pS2 Protein, EGFR and Cathepsin D in Association with Established Prognostic Factors in

Early Recurrence of Breast Cancer

Schmidt, R.; Sorger, D,; Walter, E; Schönfelder, M; Preiss, R. (Leipzig)

Letters to the Editors

181 Neumaier, M.; Gerhard, M. (Hamburg); Juhl, H. (Kiel) concerning:

Pantel K, Riethmüller G: Methods for Detection of Micrometastatic Carcinoma Cells in Bone

Marrow, Blood and Lymph Nodes

Continued on page 112

Übersichtsarbeiten 
114 Immuntherapie von Tumoren mit bispezifischen Anti-körpern

Hartmann, E; Renner, G; Pfreundschuh, M. (Homburg/Saar)

119 Neuroendokrine Tumoren des gastroenteropankreati-schen Systems: I. Aktuelle Diagnostik

Scherübl, H.; Faiss, S.; Zimmer, T.; Riecken, E.-O.; Wiedenmann, B. (Berlin)

126 Biochemische Marker des Knochenumbaus

Engler, H.; Thürlimann, B.; Riesen, W.F. (St. Gallen)

132 Wie beeinflußt die Ernährung die Entwicklung des Kolonkarzinoms? Wechselwirkung zwischen genetischen Veränderungen und Nährstoffen bei der Deregulation des Wachstums Marian, B. (Wien)

Originalarbeiten

140 Adjuvante Behandlung mit Levamisol und 5-Fluoroura-cil bei Patienten nach kurativ reseziertem Kolonkarzi-nom im Stadium Dukes C (TNM HI): Mehr Nachteile als Vorteile

Wassner, A.; Heidemann, E. (Stuttgart)

147 Kombinierte lokoregionale und systemische adjuvante Chemotherapie des

Rektumkarzinoms Stadium I und II

Kornek, G.V. (Wien); Depisch, D (Wr. Neustadt); Salem, G (St. Pölten); Karall, M. (Wr.

Neustadt); Rohrbacher, M. (St. Pölten); Scheithauer, W (Wien)

153 Phase-I-Studie zur Dosiseskalation von intravenösem Treosulfan bei therapierefraktären

Karzinomen

Harstrick, A.; Wilke, H.; Eberhardt, W.; Klaassen, U; Strumberg, D.;

Korn, M.; Scheulen, M.E. (Essen); Baumgart, J. (Hamburg); Seeber, S. (Essen)

159 Behandlung metastasierter Weichteilsarkome des Er-wachsenen mit einer Kombination aus Doxorubicin und Ifosfamid: Verbessert G-CSF die hämatologische Ver-träglichkeit?

Weh, H.J.; de Wit, M.; Zornig, C; Hossfeld, D.K. (Hamburg)

164 Plazebokontrollierte Studie von Medroxyprogesteron-azetat bei gastrointestinalen

Malignomen und Kachexie

Kornek, G.V; Schenk, E.; Ludwig, H.; Hejna, M.; Scheithauer, W (Wien)

170 Vergleich der klinischen Wertigkeit von CEA, CA 15-3 und MCA beim Mammakarzinom

Bremer, K; Micus, S.; Bremer, G (Bochum); Eberhard, A. (Dortmund)

176 pS2-Protein, EGFR und Cathepsin D in Verbindung mit etablierten Prognosefaktoren im

frühen Metastasie-rungs-/Rezidivierungsgeschehen des Mammakarzinoms

Schmidt, R.; Sorger, D.; Walter, E; Schönfelder, M.; Preiss, R. (Leipzig)

Briefe an die Herausgeber

181 Neumaier, M.; Gerhard, M. (Hamburg); Juhl, H. (Kiel) zu:

Pantel K, Riethmüller G: Methods for Detection of Micrometastatic Carcinoma Cells in Bone

Marrow, Blood and Lymph Nodes

Fortsetzung aufSeite 112

Bibliographischer Hinweis: Inhaltsverzeichnisse dieser Zeitschrift erscheinen regelmäßig in current contents ${ }^{\circledR}$ sowie in anderen bibliographischen Diensten.

Contents a Inhalt

ONKOLOGIE

Vol. 19, Issue 2, April 1996

Vol. 19, Heft 2, April 1996

182 Mistletoe in Oncology: Facts not Fiction!

Beuth, J. (Köln) concerning: 
Gabius HJ, Gabius S: Mistletoe in Oncology: Fact or Fiction?

182 Mistellektin in der Onkologie: Tatsachen nicht Fiktion!

Beuth, J. (Köln) zu:

Gabius HJ, Gabius S: Mistletoe in Oncology: Fact or Fiction?

Oncological Education

184 Randomization and Patient's Information

Clinical Information

192 Civil Liability in Radiation Therapy -

A Study of German and Austrian Judicature

Resch-Holeczke, A.; Ofner, H.; Haverkamp, U.; Potter, R. (Wien)

198 Book Reviews

200 Industrial Forum

Onkologische Fortbildung 184 Randomisation und Patientenaufklärung

Klinische Information

192 Zivilrechtliche Haftung in der Strahlentherapie -

Eine Untersuchung der deutschen und österreichischen Judikatur

Resch-Holeczke, A.; Ofner, H.; Haverkamp, U.; Potter, R. (Wien)

198 Buchbesprechungen 200 Industrieforum

201 Reports of Oncological Societies

109 Imprint

202 Instructions to Authors

201 Mitteilungen onkologischer Gesellschaften

109 Impressum

Contents of Next Issues

Review Articles Neuroendocrine Tumors of the Gastroenteropancreatic System:

II. Therapeutic Advances

Scherübl, H.; Buhr, H.; Faiss, S.; Zimmer, T.; Riecken, E.O.; Wiedenmann, B. (Berlin)

Adjuvant and Induction Chemotherapies in Non-Small-Cell Lung Cancer

Pirker, R.; Zöchbauer, S.; Krajnik, G.; Salzer, G.M.; Eckersberger, F; Huber, H.

(Wien/Innsbruck)

A Rational Approach to the Therapy of Cutaneous T-Cell Lym-phomas

Dummer, R.; Häffner, A.C.; Hess, M.; Burg, G. (Zurich)

A Long Way from Definition of the Molecular Basis to Benefit in the Clinical Management of

Ewing Tumors

Kovar, H.; Zoubek, A.; Gadner, H. (Wien)

Short Review

Osteosarcoma of the Adult

Tunn

Clinical Information

Brückenpflegeteam am Klinikum Mannheim

Gramlich 